\title{
Medical science must address health disparities amongst different ethnic groups
}

\author{
COVID-19 vaccine hesitancy amongst Black, Asian and minority ethnic (BAME) groups has recently been well \\ observed, and is symptomatic of wider health inequalities. An approach that unites insights from sociology and \\ medicine is the only way to address this pressing issue.
}

\section{Norris E. Igbineweka, Nomathamsanqa Tshuma and Noémi B. A. Roy}

U K government data has recently shown that Black ethnic groups have the lowest vaccine uptake amongst each vaccination cohort for COVID-19 vaccinations ${ }^{1}$. For example, 95.0\% of white British individuals of over 70 years of age received a vaccine between 8 December 2020 and 12 April 2021; by comparison, vaccine uptake amongst Black African individuals of the same age was $71.1 \%$ within the same time frame ${ }^{1}$. Previous investigations have shown vaccine uptake and health outcomes can vary with ethnicity ${ }^{2}$. To address this disparity, we require a refined understanding of race and ethnicity at micro- and macro-scales, and how these complexities fit within the world at large to create different outcomes.

The view that taking into account differences between groups and individuals benefits all members of society is not new. It was poignantly expressed by Rudolf Virchow in 1848. Reporting on a devastating typhus epidemic in Upper Silesia, the young pathologist from Berlin wrote that "Medicine is a social science, and politics is nothing but medicine on a large scale" ${ }^{3}$. Virchow explained that the science of human beings requires us to delineate problems and apply theory-derived solutions. He considered action without understanding of societal ills as ineffective, and scientific knowledge without action as not genuine ${ }^{3}$.

Ethnicity is the categorization of identities based on cultural characteristics, through attributes such as shared history, language or religion ${ }^{4}$. Similar to race (which is now widely understood to be a social rather than biological construct), ethnic categorization is fraught with inaccuracies and can underestimate the influence of individual circumstances and personal experience on identity. However, to address health disparities among ethnic groups in the UK (in particular, BAME groups), creating health information that targets specific ethnic groups may be a necessary, crude first step ${ }^{2}$.
In the context of vaccine uptake, using ethnicity-targeted health information may be a simple intervention that could make a difference in the decisions of parents as to whether their children should be vaccinated, because it signals to the parents that some specific characteristics of the child have been considered ${ }^{2}$. Despite warranted caution against using BAME as a description of identity, this raises the question of what other steps medical scientists can take to better serve different ethnic groups.

If our goal as medical scientists is to usher a new dawn of personalized medicine, then seeking a better understanding of these nuances is important.

\section{Burgeoning influence of social media} The complexities of reaching different groups have been amplified by the growing influence of social media, which entails a substitution of traditional science journalism with online social media platforms s $^{5}$.

Although these platforms give space to creativity, they can also operate unregulated and without accreditation, cultivating environments for misinformation ${ }^{5}$. Social media platforms take advantage of human confirmation bias, using sophisticated algorithms specifically designed to present recurrent flows of information that reinforce their viewpoints ${ }^{5}$. To understand what these mechanisms mean for the healthcare decisions of members of BAME communities, and in particular vaccination uptake, we require detailed studies into why some social media usage patterns can vary amongst ethnicities.

There is evidence to suggest that culturally tailored interventions can contribute to the improvement of healthcare outcomes for minority ethnic groups and improve patients' satisfaction with care $^{6}$. There are also data that support the effectiveness of social media and web-based interventions in improving health in minority ethnic groups, ranging from weight management to asthma and diabetes ${ }^{6}$.
However, some results for culturally tailored interventions can have mixed outcomes, and many practical considerations need to be taken into account to increase the likelihood of success ${ }^{6}$. The burgeoning influence of social media could nevertheless provide novel implications for how scientists and clinicians (especially those identifying as being from BAME communities) could use these platforms. It is also clear that effective design and implementation of these interventions requires an increasingly culturally sensitive approach.

\section{Inequalities in medical science} In 2020, racism, and stark racial and ethnic inequalities, in medicine became particularly prominent as the COVID-19 pandemic exposed the larger societal ill of structural inequalities. Akin to poverty, racism is a marker of the state of our global health and well-being. Longstanding apathy in society at large towards racism has meant we have had to keep revisiting the issue repetitively, like an exam one keeps failing and retaking. Knowledge of racial and ethnic inequality is not just simply a rehashing of an uncomfortable history, but is rather the drawing of lessons from historical context with a view to collective action for change. Moreover, representation in medical scientific research matters in terms of overcoming the effects of structural racism in medical treatment and research. Whether it is amongst researchers themselves or the populations we are investigating, a balance, blend and synthesis of perspectives is vital.

\section{Health inequalities}

Colonialism and past medical research abuses $^{7}$ have not only led to race- and ethnicity-related health inequalities, but have also caused deep mistrust of healthcare systems within some communities ${ }^{7}$ In present-day society, racial and ethnic inequalities are still pervasive in areas such as maternal and child health ${ }^{7}$. 


\section{Box 1 | Addressing health disparities through changes in policy and research}

\section{Recommendations}

Develop culturally tailored

communication and engagement around

health issues.

Fund studies in healthcare

decision-making and social

media use in underrepresented

communities.

Investigate the ongoing effects

of historical burden in marginalized

groups.

Support grassroots and policy strategies to authentically restore trust and build confidence with people from minority ethnic groups.
Support anti-racism, investigate racial inequality and implement tangible change.

Foster a culture of prioritizing and sustaining diversity throughout the biomedical workforce; cultivate the careers of young scientists.

Support medical scientists from Black, Asian and minority ethnic (BAME) backgrounds, increasing visibility and representation through role models.

Improve inclusion on selection panels, editorial boards and executive boards to redress the imbalance on grant award statistics and publications for BAME researchers.
Although breakthroughs in medical science, such as antibiotics, insulin and vaccines, have boosted global life expectancy over the past century, we have also seen an increase in both health and societal disparities $^{8}$. The COVID-19 pandemic has exacerbated economic inequalities, in that those with highest deprivation rates have almost double risk of death from COVID$19^{8}$. It is, therefore, no surprise the WHO Commission on Social Determinants of Health declared that "social injustice is killing people on a grand scale" ${ }^{8}$. The 'colour-blind' approach of the UK to COVID-19 has also been purported to be putting minority ethnic groups at higher risk of illness and death'.

COVID-19 vaccination hesitancy amongst BAME communities reflects a microcosm of the dynamics of wider health inequalities. Minority racial and ethnic groups are likely to suffer from substantial health comorbidities. Residential segregation, which is also rising in the $\mathrm{UK}$, is an important factor for ethnic differences in socioeconomic status ${ }^{10}$. This segregation influences health negatively, owing to a lower quality of education, fewer employment opportunities and reduced access to health-promoting resources ${ }^{10}$. The concentration of poverty in these areas can lead to acute and chronic stressors, and increased exposure to undesirable social and environmental conditions ${ }^{10}$.

\section{Fostering inclusion in health research}

Within medical research, there has been a lack of inclusion of minority groups for a myriad of reasons, such as exclusion by researchers, socioeconomic factors, limited topic-related funding awarded and low representation of researchers from some racial and minority ethnic groups ${ }^{8}$. Consequently, many biomedical health services research fail to understand the contextual and somewhat kaleidoscopic nature of ethnicity and rely on insufficient, evidence-free biological or cultural explanations for health patterns.

The dynamics of wider health inequalities have pervaded medical genetics, where initial underrepresentation of genetic data from African individuals caused the search for human genetic variation to suffer ${ }^{11}$. It is now clear that much of human genetic variation originated in Africa, and that this region is the most genetically diverse; the recent concerted inclusion of data from individuals of African origin has yielded important scientific insights ${ }^{11}$. Substantial disparities in research funding are also apparent. For example, in the USA, cystic fibrosis (which is a monogenic disorder that predominantly affects white individuals) receives $7-11 \times$ the amount of research funding per patient as compared to sickle cell disease (which is a monogenic disorder that predominantly affects individuals of Black heritage), despite sickle cell disease being $3 \times$ more prevalent than cystic fibrosis ${ }^{12}$. Landmark scientific innovation in gene editing and its application in sickle cell disease was welcomed by the medical community with optimism. However, its cost (US $\$ 1$ million per patient ${ }^{13}$ ) will most probably preclude its use in Africa where the disease burden is greatest. These challenges are now being addressed through collaborations such as that between Novartis and the Gates Foundation ${ }^{13}$.

\section{Eliminating career inequalities}

There remain inequalities within medicine and science careers: students from Black heritage and those from the most deprived communities are consistently underrepresented, as compared to the make-up of the wider population. These students are less likely to obtain high grades, be admitted to 'prestigious' universities, undertake further study or have career satisfaction ${ }^{14}$. Opportunities to gain research experiences, degrees, publications and positions of responsibility based on postgraduate selection are additionally burdensome, owing to complex socioeconomic factors ${ }^{8,14}$. Racially biased microaggressions have led to further undermining and internalized trauma during medical training. A recent paper illustrated that demographically underrepresented students innovated at greater rates than majority students, but that their novel contributions were discounted and less likely to earn influential academic positions ${ }^{15}$. By contrast, Dr Anthony Fauci (director of the National Institute of Allergy and Infectious Diseases and the Chief Medical Advisor to the US president) provided an excellent example of highlighting the scientific contribution of a Black researcher when he underscored the role of Dr Kizzmekia Corbett (an African-American viral immunologist) in the development of the Moderna vaccine.

\section{A call for action}

We have made huge medical scientific advances in cancer treatment, genome-editing technology and rapid vaccine development, to name a few. However, in societal terms, it seems we struggle to move with the same vigour that characterizes progress made by these biological and technological advances. Concepts of race were amplified in the 19th century alongside evolution and eugenics, which subscribed to the dogma that eradication of some genetic traits would allow for the advancement of others ${ }^{3}$. This provided a purported 'scientific' basis to justify colonization and the differential treatment of human beings ${ }^{4}$.

It is paramount we remove historical shackles and implement change, adopting inclusivity and diversity both in biological and sociological terms. Here, we offer some initial recommendations (Box 1). Medical science has a unique opportunity to lead because - if done right - it is the very definition of science, providing 'truth' through knowledge of the natural and social world using systematic methodology based on evidence. An anti-racist approach is not 'colour-blind'. Instead, a better discourse around issues of ethnicity, social media and their interrelationship with health may allow for an ameliorating course in which effective medical scientific research engages beneficially with individuals from all walks of life. Being perceptive to these factors can help to bring about a more 'complete 
science', enhancing our shared human experience. Vaccination and treatment disparities in the context of COVID-19 make this fundamental task ever more urgent.

Norris E. Igbineweka (D) 1,2区 Nomathamsanqa Tshuma ${ }^{3}$ and Noémi B. A. Roy (D) 4

${ }^{1}$ Centre for Haematology, Department of Immunology \& Inflammation, Imperial College London, London, UK. ${ }^{2}$ Haematology Department, King's College Hospital NHS Foundation Trust, London, UK. ${ }^{3} T$ The Royal London Hospital, Bart's Health NHS Trust, London, UK. ${ }^{4}$ Oxford University Hospitals NHS Foundation Trust and University of Oxford, John Radcliffe Hospital, Oxford, UK.

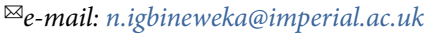

Published online: 22 November 2021

https://doi.org/10.1038/s41562-021-01240-3

References

1. Racial Disparities Unit. Third Quarterly Report on Progress to Address COVID-19 Health Inequalities. gov.uk, https://go.nature. com/3bkMZw6 (2021)

2. Forster, A. S. et al. J. Epidemiol. Community Health $\mathbf{7 1}$ 544-549 (2017).

3. Mackenbach, J. P. J. Epidemiol. Community Health 63, 181-184 (2009)

4. Smelser, N. J. \& Baltes P. B. (eds) International Encyclopedia of Social \& Behavioral Sciences, 1st edn (Pergamon, 2021).

5. Xenos, M. A. in The Oxford Handbook of the Science of Science Communication (eds. Jamieson, K. H. et al.) 283-290 (Oxford Univ. Press, 2017)

6. Armaou, M., Araviaki, E. \& Musikanski, L. Int. J. Community Well-Being 3, 193-221 (2019).

7. Bajaj, S. S. \& Stanford, F. C. N. Engl. J. Med. 384, e12 (2021).

8. FitzPatrick, M. E., Badu-Boateng, C., Huntley, C. \& Morgan, C. Future Healthc. J. 8, 12-18 (2021)

9. Osama, T., Razai, M. S. \& Majeed, A. J. R. Soc. Med. 114 240-243 (2021)
10. Harris, R., Johnston, R. \& Manley, D. Ethnicities 17, 320-349 (2017).

11. Bentley, A. R., Callier, S. L. \& Rotimi, C. N. npj Genomic Med. 5, $5(2020)$

12. Power-Hays, A. \& McGann, P. T. N. Engl. J. Med. $\mathbf{3 8 3}$, 1902-1903 (2020)

13. Novartis. Novartis and the Bill \& Melinda Gates Foundation collaborate to discover and develop an accessible in vivo gene therapy for sickle cell disease. novartis.com, https://go.nature. com/3pGsBho (2021)

14. Medical Schools Council Selection Alliance. Selection Alliance 2018 Report. An Update on the Medical Schools Council's Work in Selection and Widening Participation (Medical Schools Council, 2018)

15. Hofstra, B. et al. Proc. Natl Acad. Sci. USA 117, 9284-9291 (2020).

Acknowledgements

We thank M. Layton (Imperial College London) for his critical comments on the manuscript.

Competing interests

The authors declare no competing interests. 